\title{
Growth and Characterization of ADP Single Crystal
}

\author{
Sunil Chaki", M. P. Deshpande, Jiten P. Tailor, Mahesh D. Chaudhary, Kanchan Mahato \\ Department of Physics, Sardar Patel University, Vallabh Vidyanagar, Gujarat, 388120, India
}

\begin{abstract}
Ammonium dihydrogen phosphate (ADP) $\left(\mathrm{NH}_{4} \mathrm{H}_{2} \mathrm{PO}_{4}\right)$ single crystals were grown by gel method using sodium metasilicate (SMS). The X-ray diffraction analysis of the as-grown ADP crystals showed that it possess tetragonal structure having lattice parameters $\mathrm{a}=7.502^{\circ} \mathrm{A}$ and $\mathrm{c}=7.554^{\circ} \mathrm{A}$. The Fourier transform infrared spectroscopy (FTIR) of as-grown ADP crystal taken between wave-number 400 to $4000 \mathrm{~cm}^{-1}$ showed peaks due to vibration and stretching of functional group $\mathrm{O}-\mathrm{N}=\mathrm{P}$ and $-\mathrm{ONO}_{2}$ in 485 to $902 \mathrm{~cm}^{-1}, \mathrm{P}=\mathrm{O}$ and $\mathrm{O}-\mathrm{H}$ in 1076 to $1544 \mathrm{~cm}^{-1}$ and $\mathrm{O}-\mathrm{H}$ and $\mathrm{N}-\mathrm{H}$ in 2400 to $3371 \mathrm{~cm}^{-1}$ range. The UV-Vis-NIR spectroscopy of ADP crystal showed direct optical bandgap of $4.99 \mathrm{eV}$ and indirect optical bandgap of $4.12 \mathrm{eV}$. The optical microscopy employed to study the surface microstructure showed hillocks on the as-grown surfaces arising due to local increased supersaturation. The thermal properties of the as-grown ADP crystals were studied by thermogravimetric analysis (TGA). The thermal activation energy determined from the TGA curve using Broido, Piloyan-Novikova (PN) and Coats Redfern (CR) relations were in good agreement with each other. The obtained results are discussed in details.
\end{abstract}

Keywords Gel growth, ADP, single crystals, XRD, optical characterization, thermal study

\section{Introduction}

Ammonium dihydrogen phosphate (ADP) $\left(\mathrm{NH}_{4}\right) \mathrm{H}_{2} \mathrm{PO}_{4}$ is an interesting material with varied application as a piezoelectric material in transducer devices, nonlinear optics (NLO), electro-optics, and as monochromators for X-ray fluorescence analysis[1-6]. ADP was among the first material that were used and exploited for their non-linear optical (NLO) and electro-optic (EO) properties[7]. They are widely used as the second, third and fourth harmonic generator in Nd:YAG and Nd:YLF lasers. Studies on ADP crystals still attract interest because of their unique nonlinear optical, dielectric and antiferroelectric properties[8-10]. In recent time, R. Kripal et al[1] studied electron paramagnetic resonance (EPR) spectra of copper doped ADP. Whereas optical, mechanical, dielectric, piezoelectric and laser damage threshold studies have been carried out on mixed ADP-KDP crystal by P. Rajesh et al[11]. The literature shows that mostly the ADP crystals are grown by solution growth in which the crystal or the solution from which it is growing are in motion during growth having probability of defects. Thus the authors used gel growth method for growth of ADP crystals, in which the growth takes place without any movement. There are various types of gel like silica gel, sodium metasilicate, agar gel, gelatin gel, clay gel, soap gel, etc. Literature shows no report of ADP crystals in sodium metasilicate gel, so the authors selected sodium metasilicate as the gel material. In this paper, the authors report growth of

* Corresponding author:

sunilchaki@yahoo.co.in (Sunil Chaki)

Published online at http://journal.sapub.org/ajcmp

Copyright (C) 2012 Scientific \& Academic Publishing. All Rights Reserved
ADP single crystals by gel method and its structural, optical, thermal characterization studies.

\section{Experimental}

\subsection{Sample Preparation}

Single crystals of ammonium dihydrogen phosphate (ADP) $\left(\mathrm{NH}_{4}\right) \mathrm{H}_{2} \mathrm{PO}_{4}$ have been grown in sodium metasilicate (SMS) gel at room temperature. The apparatus used for crystallization consists of borosilicate glass tubes of length $15 \mathrm{~cm}$ and diameter $2.4 \mathrm{~cm}$ (I.D.) placed vertical in a wooden stand. An aqueous solution of tartaric acid of a particular molarity (1M, $10 \mathrm{ml}$ ) was taken in a beaker and sodium metasilicate (SMS) $(1.04 \mathrm{gm} / \mathrm{ml})$ of a particular specific gravity was added drop-wise, using a burette, constantly stirring the solution in the beaker with a view of avoiding excessive local ion concentration which may cause premature local gelling and make the final medium inhomogeneous and turbid. The gel solution with the desired value of $\mathrm{pH}$ (upto 3-5), as measured using a digital $\mathrm{pH}$ meter (model No: PHCAL, Analab Scientific Instruments Pvt. Ltd, Vadodara, India) was transferred to several test tubes, in a fixed amount, without giving a chance for the formation of air bubbles, by allowing the mixture to fall steadily alongside of the test tubes walls. Mouths of the test tubes were then closed with cotton to prevent fast evaporation from and contamination of the exposed surface of the gel. The gel was usually found to set in 10 to 15 days, depending upon the environmental temperature and its $\mathrm{pH}$. After ensuring firm gel setting, an aqueous solution of ADP $\left(\mathrm{NH}_{4}\right) \mathrm{H}_{2} \mathrm{PO}_{4}$ of a particular concentration $(1 \mathrm{M}, 10 \mathrm{ml})$ was poured over the set gel, with the help of a pipette, being allowed to fall along the wall of the test tubes 
so as to prevent the gelled surface from cracking. The resulting gel-liquid interface is then allowed to stand for 3 to 4 weeks. During this time a number of colourless good quality transparent crystals appear in the gel medium which is shown in the Figure 1.

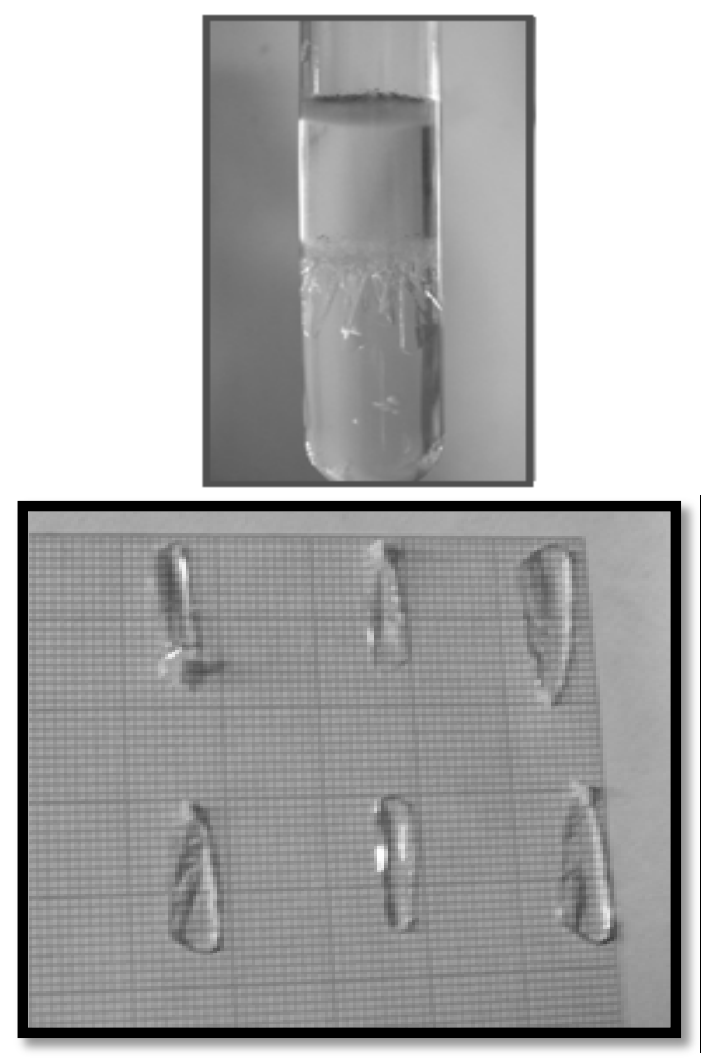

Figure 1. Photograph of transparent ADP single crystal

\subsection{Characterization of ADP Single Crystals}

The crystallographic lattice parameters of the gel grown ADP single crystals were determined using $X$-ray diffraction employing Philips X-ray diffractometer X-pert-MPD using $\mathrm{CuK}_{\alpha}$ radiation in the $2 \theta$ range of $15^{\circ}-90^{\circ}$. The FTIR spectrum was recorded in the regions $400-4000 \mathrm{~cm}^{-1}$ using a Perkin-Elmer FTIR Spectrometer [GX Spectrometer] by $\mathrm{KBr}$ pellet technique. The optical properties of as-grown ADP crystals were studied using UV-Vis-NIR spectrophotometer Perkin Elmer Lambda-19 in the range of $185 \mathrm{~nm}$ to $3200 \mathrm{~nm}$. The surfaces of the gel grown ADP crystals were examined under 'Axiotech $100 \mathrm{HD}$ ' optical microscope [Carl Ziess (India) Pvt. Ltd, Bangalore, India] for their microstructures. The thermal analysis of the single crystals of ADP was done by Thermogravimetric analyser. The thermograph (TG) was acquired from ambient $(300 \mathrm{~K})$ to $873 \mathrm{~K}$ at a heating rate of $5{ }^{\circ} \mathrm{C} / \mathrm{min}$ in inert (Nitrogen) atmosphere using a Perkin-Elmer Pyris-1 TGA.

\section{Results and Discussion}

\subsection{Structural Studies (XRD)}

The crystallographic structure and lattice parameters of gel grown ADP single crystals were determined from the X-ray diffraction pattern obtained employing X-ray diffractometer. The diffraction peaks of the XRD patterns shown in Figure 2 could be indexed as those of the ADP with tetragonal structure (JCPDS Card No.37-1479). The XRD peaks were indexed and crystallographic lattice parameters were determined by powder- $\mathrm{X}$ software. The determined lattice parameters are $\mathrm{a}=7.502^{\circ} \mathrm{A}$ and $\mathrm{c}=7.554^{\circ} \mathrm{A}$ having space group $I \overline{4} 2 d$.The lattice parameters are in good agreement with the reported values[12].

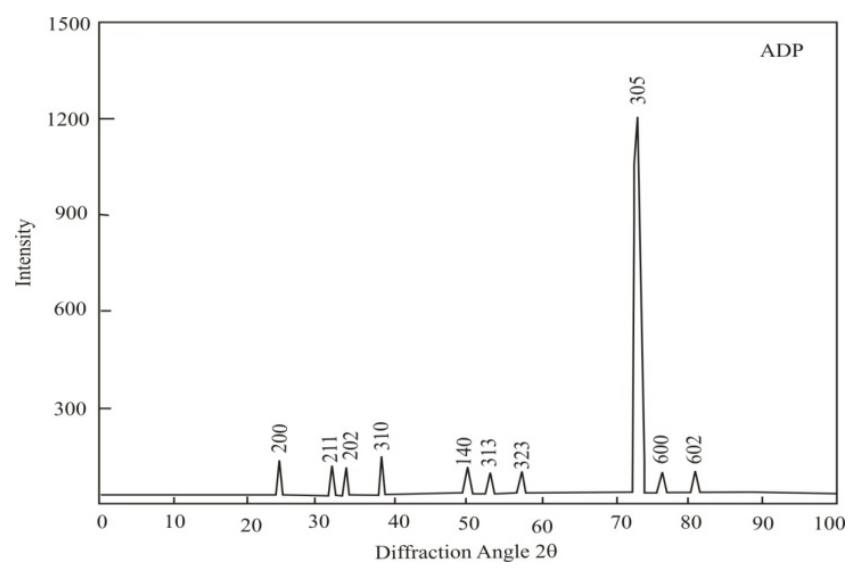

Figure 2. X-ray powder diffraction of ADP

\subsection{Fourier Transform Infrared Spectroscopy (FTIR)}

The functional groups of pure ADP crystals involved in vibration frequency have been identified using FTIR spectroscopy.

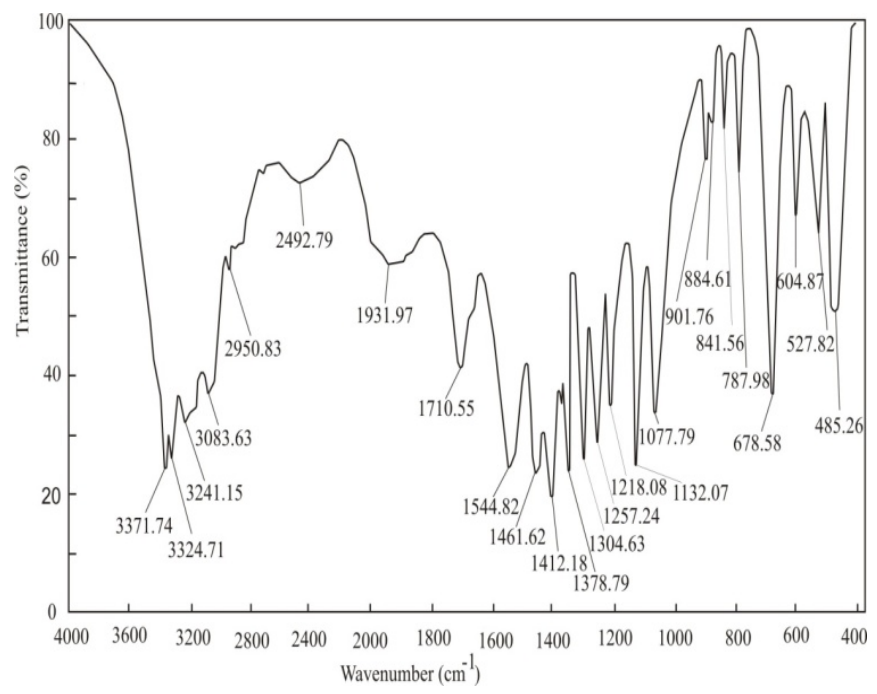

Figure 3. FTIR spectrum of ADP single crystal

The gel grown ADP FTIR spectrum was taken between wave-number 400 to $4000 \mathrm{~cm}^{-1}$, shown in Figure 3. The peaks between 485 to $902 \mathrm{~cm}^{-1}$ are due to the $\mathrm{O}-\mathrm{N}=\mathrm{P}$ and $-\mathrm{ONO}_{2}$ bond vibration in ADP crystal. Whereas $\mathrm{P}=\mathrm{O}$ and $\mathrm{O}-\mathrm{H}$ are responsible for the peaks in the wave-number range, 1076 to $1544 \mathrm{~cm}^{-1}$. In the wave-number range from 2400 to $3371 \mathrm{~cm}^{-1}$, the numbers of peaks are lesser than the above two wave-number ranges. These peaks are due to $\mathrm{O}-\mathrm{H}$ and N-H stretching[13]. 


\subsection{Optical Studies UV-Vis-NIR Spectroscopy}

The optical properties of as-grown ADP single crystals were studied using UV-Vis-NIR spectrophotometer in the range of $185 \mathrm{~nm}$ to $3200 \mathrm{~nm}$. The absorption spectrum of the as-grown ADP single crystals is shown in Figure 4.

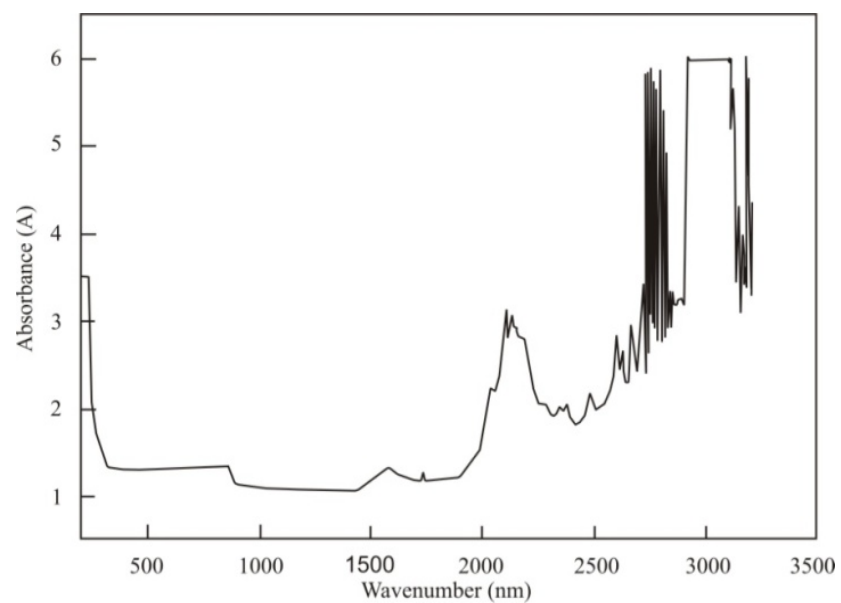

Figure 4. Absorption spectrum of ADP single crystal

The optical band gap of the ADP single crystal was determined from the absorption spectrum using the near-band edge absorption relation,

$$
(\alpha \mathrm{h} v)^{\mathrm{n}}=\mathrm{A}\left(\mathrm{h} v-\mathrm{E}_{\mathrm{g}}\right)
$$

Where A - the optical transition dependent constant, $E_{g}$ optical energy bandgap, $v$ - the frequency of incident beam, $h$ - Planck's constant, $\mathrm{n}$ - characterizes the transition. For direct allowed and forbidden transitions, $n=2$ and $2 / 3$ respectively, and $n=1 / 2$ and $1 / 3$ for indirect allowed and forbidden transitions, respectively.

The analysis of equation[1] showed that, $\mathrm{n}=2$ and $1 / 2$ fitted for the as-grown ADP single crystal samples confirming direct and indirect allowed transition. Figure 5(a) and 5(b) shows the plot of $(\alpha h v)^{2}$ against hv and $(\alpha h v)^{1 / 2}$ against $h v$ respectively for ADP crystals. The intercept of the straight line on the photon energy axis gives the direct bandgap value of $4.99 \mathrm{eV}$ and indirect bandgap value of $4.12 \mathrm{eV}$.

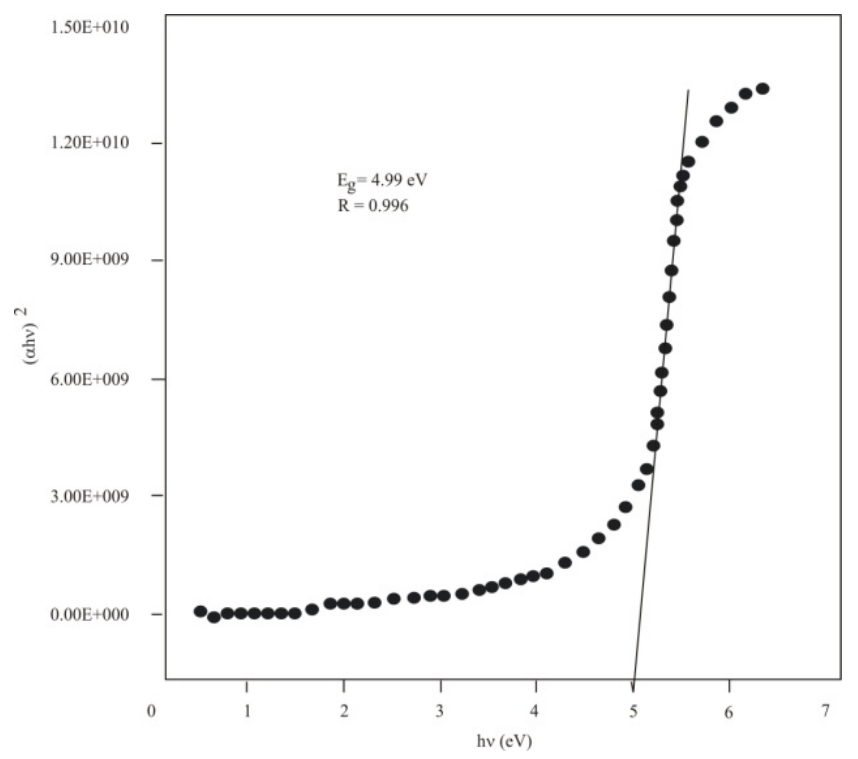

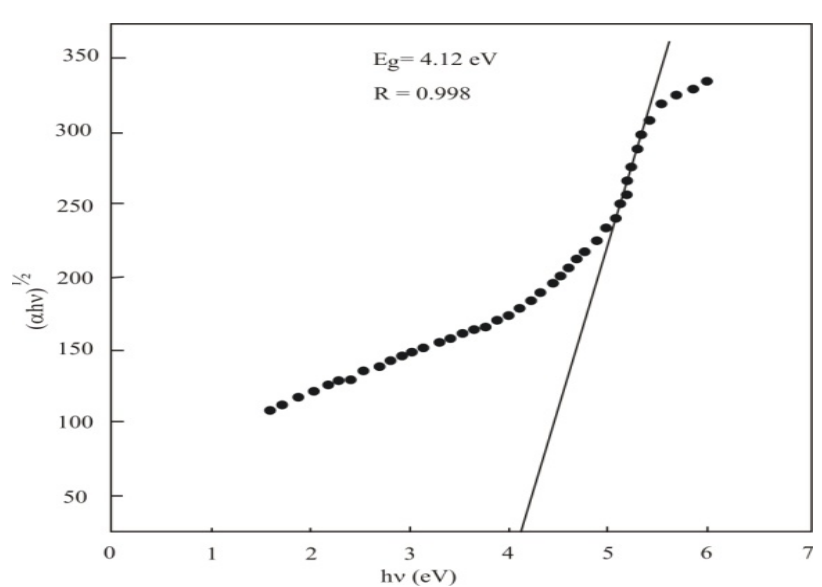

Figure 5. Plot of (a) $(\alpha h v)^{2}$ versus hv giving direct band gap (b) $(\alpha h v)^{1 / 2}$ versus $h v$ giving indirect band gap

\subsection{Optical Microscopy}

The most common features visible on the as-grown surfaces of gel grown ADP were hillocks as shown in Figure 6.

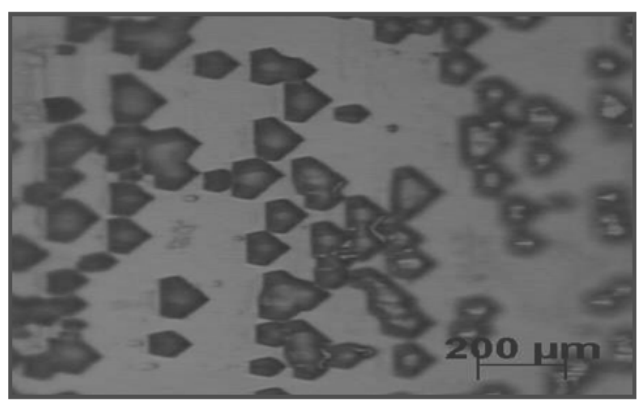

Figure 6. Optical micrograph of gel grown ADP single crystals

There is no satisfactory interpretation of such large nondislocation hillocks in the literature. The only explanation available is that, they are produced by the two dimensional nucleation at some points on the surface due to impurities in the growth environments and when the supersaturation at some points on the growing surface of the crystal is higher than that of the rest of the surface. Due to this local increased supersaturation, these points act as centres of repeated two-dimensional nucleation for growth fronts, which spread and pile upon one another to produce the hillocks.

\subsection{Thermogravimetric Analysis}

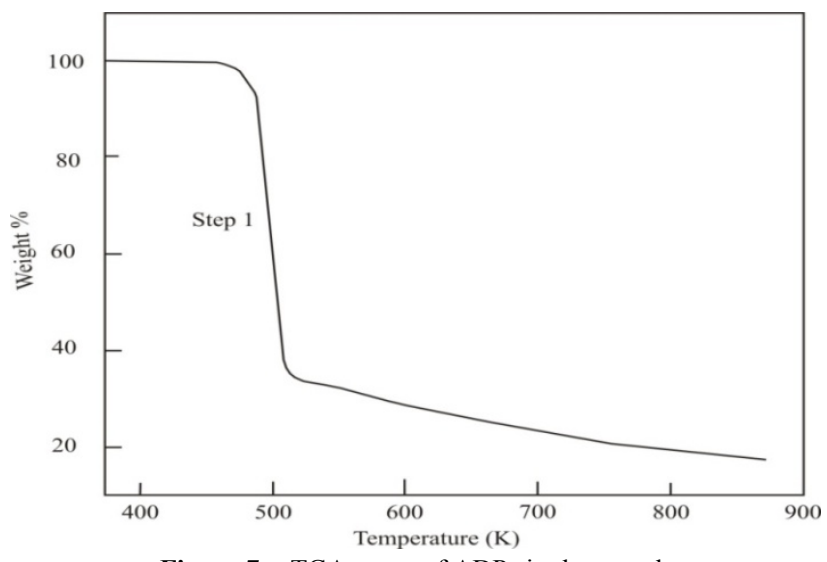

Figure 7. TGA curve of ADP single crystal 
The thermogravimetric curve obtained for gel grown ADP in inert atmosphere is shown in Figure 7. The curve clearly shows that the ADP crystal is stable between the temperature range of ambient to $473 \mathrm{~K}$ (the melting point of ADP). Above $473 \mathrm{~K}$ to $873 \mathrm{~K}$, the weight loss in total is of $\sim 80 \%$. The maximum weight loss in one step ( $473 \mathrm{~K}$ to $523 \mathrm{~K}$ ) is $\sim 63 \%$. The activation energy calculation using this weight loss in temperature range $473 \mathrm{~K}$ to $523 \mathrm{~K}$ was carried out using Broido, Piloyan-Novikova (P-N) and Coats-Redfern (C-R) relations[14]. The plot of Broido, Piloyan-Novikova (P-N) and Coats-Redfern (C-R) is shown in Figure 8. The activation energy obtained from the plots of these three relations is tabulated in Table 1. The values are in good agreement with each other.
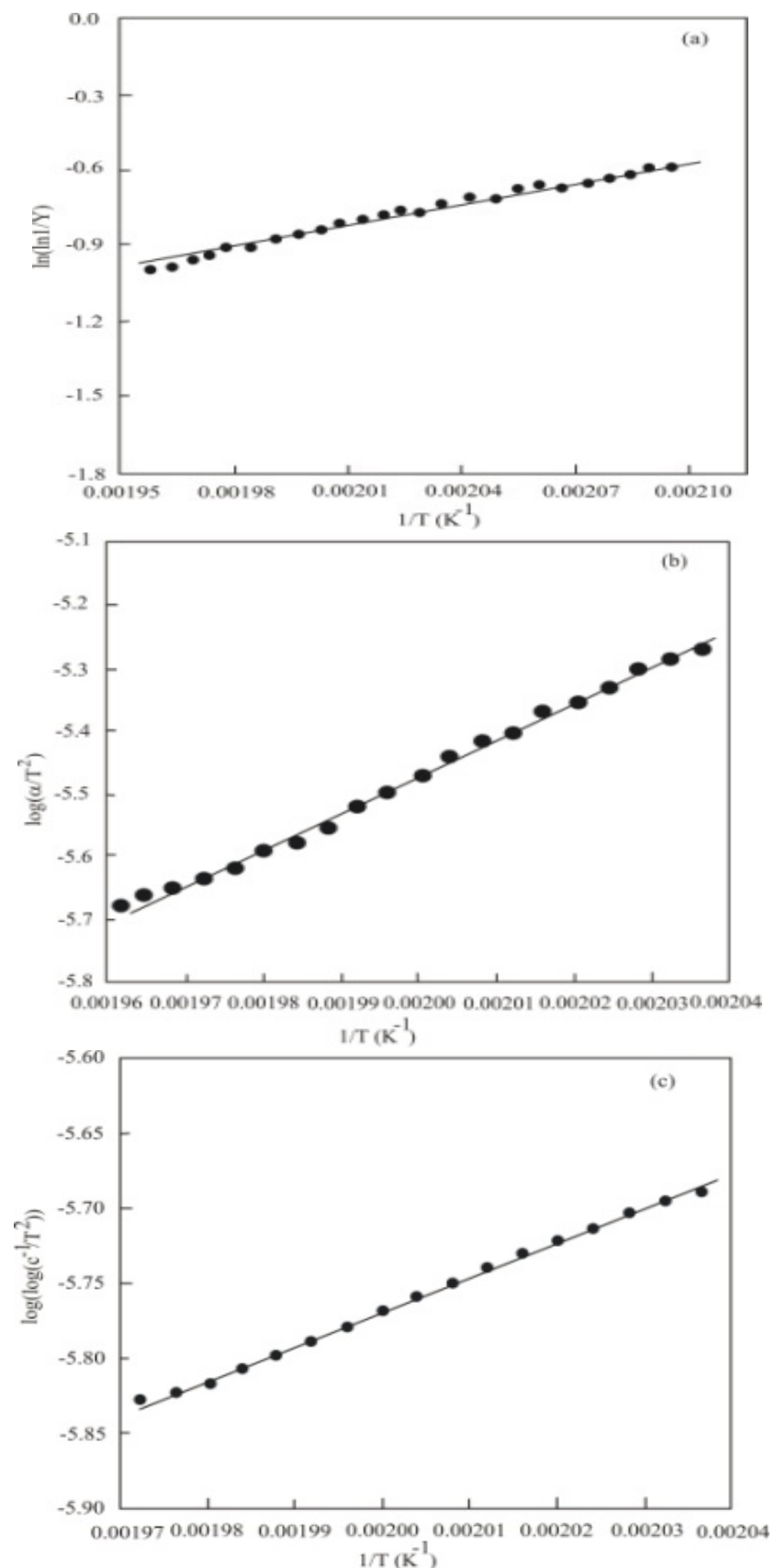

Figure 8. Plot of (a) Broido relation (b) Piloyan-Novikova (P-N) relation (c) Coats-Redfern (C-R) relation
Table 1. Kinetic parameters evaluated from the non-mechanistic equations for gel grown ADP crystals

\begin{tabular}{|c|c|c|c|}
\hline Method & $\begin{array}{c}\text { Broido } \\
\text { relation }\end{array}$ & $\begin{array}{c}\text { Piloyan-Novikova } \\
\text { (P-N) relation }\end{array}$ & $\begin{array}{c}\text { Coats-Redfern } \\
\text { (C-R) relation }\end{array}$ \\
\hline $\begin{array}{c}\text { Activation } \\
\text { energy } \mathrm{E}_{\mathrm{a}} \text { in } \mathrm{eV}\end{array}$ & 0.354 & 0.327 & 0.297 \\
\hline
\end{tabular}

\section{Conclusions}

Good quality transperent single crystals of ammonium dihydrogen phosphate (ADP) $\left(\begin{array}{llll}\mathrm{NH}_{4} & \mathrm{H}_{2} & \mathrm{PO}_{4}\end{array}\right)$ have been grown in sodium metasilicate (SMS) gel at room temperature. The X-ray diffraction pattern of the gel grown ADP showed the crystals possess tetragonal structure with lattice parameters in good agreement with the reported data (JCPDS Card No.37-1479). The functional groups of ADP crystals involved in vibration frequency were identified using FTIR. The optical bandgap values determined from the optical absorbance study of the crystals showed direct bandgap value of $4.99 \mathrm{eV}$ and indirect bandgap value of $4.12 \mathrm{eV}$. The most common features visible on the as-grown surfaces of gel grown ADP were hillocks, this was due to local increased supersaturation. The thermogravimetric curve obtained for gel grown ADP in inert atmosphere in temperature range ambient to $873 \mathrm{~K}$, shows that the gel grown ADP crystal is stable between room temperature and $473 \mathrm{~K}$ (the melting point of ADP). Above $473 \mathrm{~K}$ to $873 \mathrm{~K}$, the weight loss is $80 \%$. The maximum weight loss of $\sim 63 \%$ in one step is in temperature range $473 \mathrm{~K}$ to $523 \mathrm{~K}$. The activation energy value calculation in temperature range $473 \mathrm{~K}$ to $523 \mathrm{~K}$ using Broido, Piloyan-Novikova (P-N) and Coats-Redfern (C-R) relations are in good agreement with each other.

\section{REFERENCES}

[1] Ram Kripal, Santwana Shukla, Prashant Dwivedi, EPR and optical studies of $\mathrm{Cu}^{2+}$ doped ammonium dihydrogen phosphate single crystals, Physica B 407 (2012) 656-663.

[2] P. Rajesh, K. Boopathi, P. Ramasamy, Investigations on the solubility, growth, structural, optical, mechanical, dielectric and SHG behaviour of ammonium acetate doped ammonium dihydrogen phosphate crystals, J Crystal Growth 318 (2011) 751-756.

[3] K. Srinivasan, A. Cantoni, G. Bocelli, Compositional dependence of morphology and lattice parameters during growth of $\mathrm{K}_{1-\mathrm{x}}\left(\mathrm{NH}_{4}\right)_{\mathrm{x}} \mathrm{H}_{2} \mathrm{PO}_{4}$ mixed crystals, Cryst. Res. Technol. 45(7) (2010) 737-746.

[4] R. Ananda Kumari, Growth and characterization of NLO crystal, Ind. J. Pure \& Appl. Phys. 47 (2009) 369-371.

[5] Y. Asakuma, Q. Li, H. M. Ang, M. Tade, K. Maeda, K. Fukui, A study of growth mechanism of KDP and ADP by means of quantum chemistry, Appl. Surface Sci. 254(15) (2008) 4524-4530.

[6] Xiue Ren, Dongli Xu, Dongfeng Xue, Crystal growth of KDP, ADP, and KADP, J Crystal Growth 310 (2008) 2005-2009. 
[7] Dongfeng Xue and Henryk Ratajczak, Effect of hydrogen bonds on physical properties of ammonium dihydrogenphosphate crystals, J Molecular Structure: Theochem. 716 (2005) 207-210.

[8] K. Sethuraman, R. Ramesh Babu, R. Gopalkrishnan, P. Ramasamy, Unidirectional growth of $\left\langle\begin{array}{llll}1 & 1 & 0\end{array}\right\rangle$ ammonium dihydrogen orthophosphate single crystal by Sankaranarayanan-Ramasamy method, J Crystal Growth 294 (2006) 349-352.

[9] A. P. Voronov, V. I. Salo, V. M. Puzikov, G. N. Babenko, A. D. Roshal, V. F. Tkachenko, Hybrid organic-inorganic crystals based on ammonium dihydrogen phosphate and ammonium salicylate, J Crystal Growth 335 (2011) 84-89.

[10] P. Rajesh, P. Ramasamy, Binay Kumar, G. Bhagavannarayana, Effect of cobalt and DL-malic acid on the growth rate, crystalline perfection, optical, mechanical, dielectric, piezoelectric properties and SHG efficiency of ADP single crystals, Physica B 405 (2010) 2401-2406.

[11] P. Rajesh, P. Ramasamy, G. Bhagavannarayana, Growth of ADP-KDP mixed crystal and its optical, mechanical, dielectric, piezoelectric and laser damage threshold studies, J. Crystal Growth, doi:10.1016/j.jcrysgro.2011.10.036.

[12] P. Shenoy, K. V. Bangera, G. K. Shivakumar, Growth and thermal studies on pure ADP, KDP and mixed $\mathrm{K}_{1-\mathrm{x}}\left(\mathrm{NH}_{4}\right)_{\mathrm{x}} \mathrm{H}_{2} \mathrm{PO}_{4}$ crystals, Cryst. Res. Technol. 45 (2010) 825-829.

[13] Russel S Drago, Physical Method in Inorganic Chemistry (Chapter 7), East-West Press Pvt. Ltd, New Delhi (1968).

[14] Sunil. H. Chaki, Thermal decomposition studies of $\mathrm{CuInS}_{2}$, Front. Mater. Sci .China, 2(3) (2008) 322-325. 